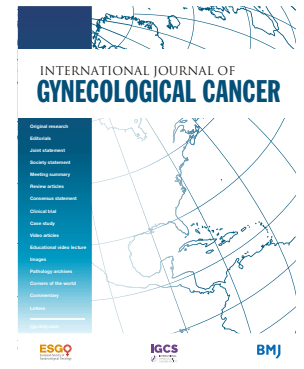

${ }^{1}$ Gynecologic Oncology, Nairi Medical Center, Yerevan,

Armenia

${ }^{2}$ Obstetrics and Gynecology, National Institute of Health, Yerevan, Armenia

\section{Correspondence to}

Professor Artem Stepanyan, Gynecologic Oncology, Nairi Medical Center, Yerevan 0015 Armenia; stepanyan.artem@ gmail.com

Accepted 19 April 2021

\title{
Right upper quadrant peritonectomy. Answering frequently asked questions
}

\author{
Artem Stepanyan (D) , ${ }^{1,2}$ Zhirayr Malakyan, ${ }^{1}$ Areg Alaverdyan, ${ }^{1}$ Hasmik Davtyan, \\ Tatevik Hovhannisyan ${ }^{1}$
}

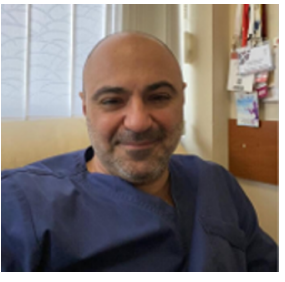

\section{Artem Stepanyan \\ ${ }^{1}$ Gynecologic Oncology, Nairi Medical Center, Yerevan, Armenia \\ ${ }^{2}$ Obstetrics and Gynecology, National Institute of Health, Yerevan, Armenia}

Biography: Dr Artem Stepanyan is the Head of the Gynecologic Oncology Service at NAIRIMedical Center in Yerevan, Armenia. He is also an Associate Professor at theDepartment of OB/GYN in National Institute of Health (Institute of Postgraduate MedicalEducation). The topic of his special interest is the surgery of gynecologicalmalignancies.

Removal of upper abdominal disease remains one of most challenging parts of surgery in advanced ovarian cancer. The right upper quadrant of the abdominal cavity is an anatomical region that generally is the most affected by cancer metastases in those patients. This video educational lecture is an attempt to answer the most frequently asked questions related to the technical aspects of the right upper quadrant peritonectomy procedure. Video 1 We obtained these questions from a survey conducted for fellows and early career gynecologic oncologists. The video lecture comprises the answers $(A)$ to the preceding questions $(Q)$ by graphic images and technical videos with corresponding voiceover.

- Q\#1: Does the volume of right upper quadrant disease matter?

- A\#1: (surgical video) Besides the volume the distribution of the disease rather matters. The Glisson's capsule and the coronary ligament of the liver are sometimes deeply infiltrated by the

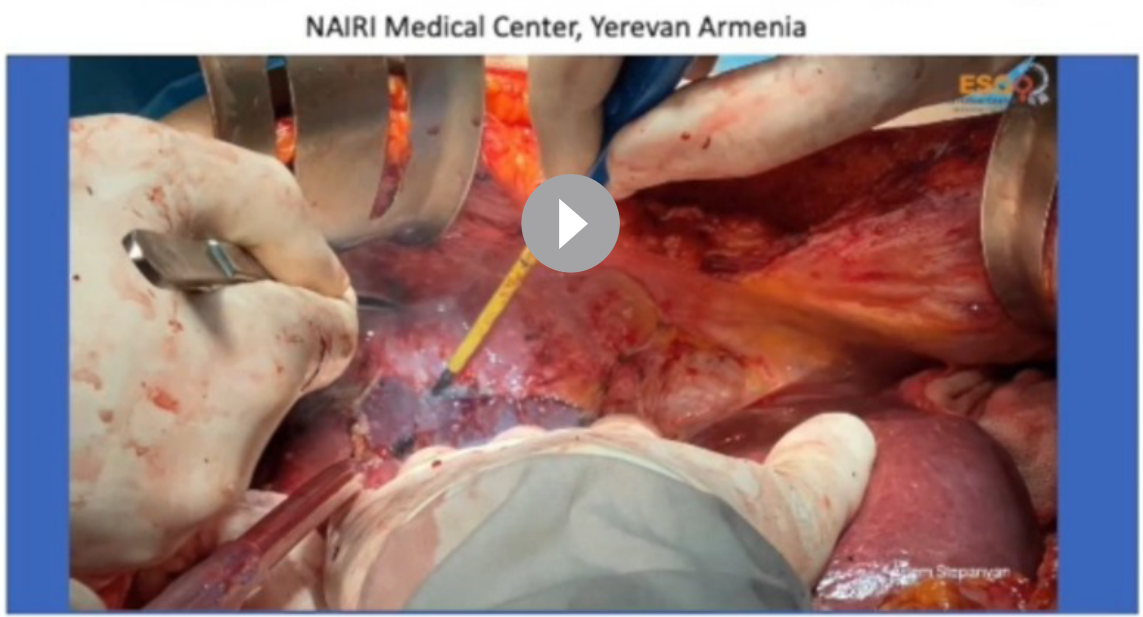
commercial re-use. See rights and permissions. Published by BMJ.

\begin{tabular}{l}
\hline To cite: Stepanyan A, \\
Malakyan Z, Alaverdyan A, \\
et al. Int J Gynecol Cancer \\
2021;31:1305-1306. \\
\hline
\end{tabular}

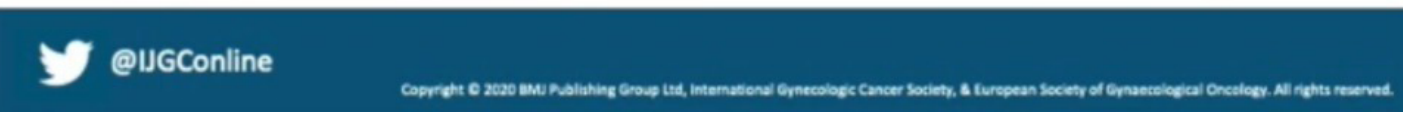




\section{Educational video lecture}

tumor metastases and good expertise is needed to localize and dissect the critical vascular landmarks.

- Q\#2: What are my critical vascular landmarks?

- A\#2: (graphic scheme) The supra-hepatic vena cava and its tributaries such as the middle, right, and left hepatic veins are the first critical vascular landmarks. The second is the infrahepatic vena cava. ${ }^{1}$

- Q\#3: Nice picture... But what does it look like in the reality?

- A\#3: (graphic image from cadaveric dissection) The area of interest is shown. The anatomic relationships between the supra-hepatic cava, the liver, the diaphragm, and the pericardium are demonstrated.

- Q\#4: How do I safely avoid the supra-hepatic vena cava?

- A\#4: (surgical video) The right coronary ligament is being incised over the supra-hepatic vena cava and the para-vascular space, located just laterally to the right hepatic vein, is entered. This gives good visual control over this vascular landmark. ${ }^{2}$

- Q\#5: How do I safely avoid the infra-hepatic vena cava?

- A\#5: (surgical video) For good access to the infra-hepatic inferior vena cava and Morrison's pouch peritoneum the liver is manually retracted upwards and cephalad, the hepatic flexure of the colon is retracted caudad, and the duodenum is retracted medially. So, the inferior portion of the coronary ligament and the infra-hepatic vena cava are being visualized. ${ }^{3}$

- Q\#6: What is the feature of the monopolar ball tip electrode that you use?

- A\#6: (graphic scheme and surgical video) We consider that the $3 \mathrm{~mm}$ ball tip monopolar electrode is small enough for fine dissection, large enough for hemostasis, and it is cheap.

- Q\#7: Are there other vascular landmarks that I need to pay attention to?
- A\#7: (surgical video). The inferior phrenic veins should be respected while performing the liver mobilization and diaphragm stripping or resection.

- Q\#8: How do I effectively handle the liver?

- A\#8: (surgical video) The technique of safe liver handling and retraction using a laparotomy pad is demonstrated.

- Q\#9: Can you please show me the entire procedure?

- A\#9: (surgical video) The technique of trans-peritoneal right upper quadrant peritonectomy and diaphragm resection is demonstrated.

We hope that this video lecture will be useful for those who are interested in advanced ovarian cancer surgery.

Contributors All authors contributed equally to this article.

Funding The authors have not declared a specific grant for this research from any funding agency in the public, commercial or not-for-profit sectors.

Competing interests None declared.

Patient consent for publication Not required.

Provenance and peer review Not commissioned; externally peer reviewed.

ORCID iD

Artem Stepanyan http://orcid.org/0000-0003-1961-0004

\section{REFERENCES}

1 Shchukin D, Lesovoy V, Garagatiy I, et al. Surgical approaches to supradiaphragmatic segment of IVC and right atrium through abdominal cavity during intravenous tumor thrombus removal. Adv Urol 2014;2014:1-9.

2 Ramirez PT, Frumovitz M. Principles of gynecologic oncology surgery. Philadelphia: Saunders/Elsevier, 2019.

3 Bristow RE, Karlan BY, Chi DS. Surgery for ovarian cancer: principles and practice. New York: Informa Healthcare, 2010. 